\title{
Expressions of TLR4, MyD88 and IRAK4 in the Skin of Chinese Brown Frog (Rana Dybowskii) During the Breeding and Pre-Hibernation Periods
}

\author{
Ao Zhang, Yue Zhang, Yuan Chen, Yuyao Shen, Xiaohang Feng, Haolin Zhang, Qiang Weng and Meiyu \\ $\mathrm{Xu}^{*}$ \\ College of Biological Science and Technology, Beijing Forestry University, China \\ *Corresponding author: Meiyu Xu, College of Biological Sciences and Biotechnology, Beijing Forestry University, China
}

\section{ARTICLE INFO}

Received: 陆 December 09, 2019

Published: 幽 December 17, 2019

Citation: Ao Zhang, Yue Zhang, Yuan Chen, Yuyao Shen, Xiaohang Feng, Haolin Zhang, Qiang Weng, Meiyu Xu. Expressions of TLR4, MyD88 and IRAK4 in the Skin of Chinese Brown Frog (Rana Dybowskii) During the Breeding and Pre-Hibernation Periods. Biomed J Sci \& Tech Res 23(5)2019. BJSTR. MS.ID.003979.

Keywords: TLR4; MyD88; IRAK4; Rana dybowskii; Skin

\begin{abstract}
Toll-Like Receptor 4 (TLR4) recruits the adaptor protein myeloid differentiation primary response gene 88 (MyD88) and Interleukin-1 Receptor-Associated Kinase 4 (IRAK4), triggering a series of immune responses. In this study, we investigated the localizations and expression levels of TLR4, MyD88 and IRAK4 in the skin of Rana dybowskii during the breeding and pre-hibernation periods. Histologically, the skin of Rana dybowskii consisted of epidermis and dermis. The epidermis consisted of stratum corneum, stratum granulosum, stratum spinosum and stratum germinativum, while the dermis was composed of homogenous gel, mucous glands and granular glands. TLR4, MyD88 and IRAK4 were immunolocalized in the epithelial and glandular cells of skin in both periods. Western blotting revealed that TLR4, MyD88 and IRAK4 were expressed in the breeding and pre-hibernation periods. Real-Time PCR showed that the mRNA expression level of MyD88 in the pre-hibernation decreased when compared with the breeding period, while the mRNA expression levels of TLR4 and IRAK4 were not significantly different between the breeding and pre-hibernation periods. These results suggested that TLR4, MyD88 and IRAK4 might participate in the skin immune system of Rana dybowskii during the breeding and pre-hibernation periods.
\end{abstract}

\section{Introduction}

Toll-Like Receptors (TLRs) are membrane-bound proteins that recognize invading organisms with Pathogen-Associated Molecular Patterns (PAMPs) and Damage-Related Molecular Patterns (DAMPs) [1]. TLRs activated by PAMP or DAMP could upregulate chemokines and inflammatory cytokines and participate in various intracellular signaling pathways to regulate the inflammatory responses (Brown et al., 2011). TLR4, the first TLR to be identified by Medzhitov in 1997 , is the only receptor that mediates the immune response through the MyD88-dependent signaling pathway and the MyD88independent signaling pathway and is a key component of the innate immune system [2]. TLR4 is expressed in epithelial cells and activated TLR4 causes activation of Nuclear factor $\kappa B$ (NF$\kappa \mathrm{B})$ through multiple downstream intracellular signals, and then synthesizes pro-allergic cytokines [3]. TLR4 recruit's adaptor proteins such as MyD88 to IRAK4 and TNF receptor associated factor 6 (TRAF6), finally induces the activation of COX2, which plays a key regulatory role in skin inflammation Sherwani (2018).

MyD88 is an adaptor protein for most TLR signaling pathways in regulation of the innate immunity [4]. Upon recruitment by TLRs, MyD88 activates NF- $\kappa B$, which leads to the subsequent transcription and expression of inflammatory genes such as TNF- $\alpha$, IL-1 and IL-6 Palti [5]. Studies have shown that MyD88-dependent signaling could exacerbate allergic inflammation, and the lack of MyD88 could protect mice with skin barrier function from spontaneous skin damage [6,7]. IRAK4 is a serine-threonine kinase that is critical in the signaling cascade and is central to MyD88-dependent signaling. IRAK4-deficiency animals completely lost their immune response to bacterial and viral challenge, which indicate that IRAK4 plays an important role in innate immunity [6]. The skin of the amphibian is directly exposed to the complex environment. As an 
organ that is in direct contact with the external environment, it is vulnerable to external factors such as predators, microorganisms, parasites and physical damage [9]. To resist the harmful effects of environmental factors, the skin of amphibians has formed a stable defense mechanism in the long-term evolution process. The main function of the skin immune system is to promote wound healing and immune tolerance [10].

The Chinese brown frog (Rana dybowskii) is distributed in highhumidity environments in China, Japan, Korea and eastern Siberia [11]. The breeding period of the Rana dybowskii is from early April to early May, and from early September to early October is the prehibernation period, it migrates to the river or pond, waiting for winter. Their skin therefore acts as the first line of defense against invading pathogens. Granular glands in the skin tissue of Rana dybowskii synthesize and secrete biologically active molecules such as antibacterial peptides and antioxidants associated with innate immunity, which contribute to epithelial defense [12]. Previous studies have shown that the expression of NF- $\kappa \mathrm{B}$, a downstream factor of the TLR signaling pathway, is present in Rana dybowskii skin, suggesting that NF- $\mathrm{B}$ may play a significant role in the skin immune system of Rana dybowskii [11]. In addition, MyD88 is expressed in various tissues of Rana dybowskii and has a conserved structure, which might be an important component of the innate immunity in Rana dybowskii [13]. In this study, we investigated the expressions of TLR4, MyD88 and IRAK4 in Rana dybowskii skin during the breeding and pre-hibernation periods to clarify the roles of TLR4, MyD88 and IRAK4 in the skin immune system of Rana dybowskii.

\section{Materials and Methods}

\section{Animals}

Thirty adult female Chinese brown frogs were obtained in April (breeding period, $\mathrm{n}=15$ ) and September (pre-hibernation, $\mathrm{n}=15$ ) from Jilin Baektu Mountain Chinese Brown Frog Breeding Farm, Jilin Province $\left(125^{\circ} 40^{\prime} \mathrm{E}-127^{\circ} 56^{\prime} \mathrm{E}, 42^{\circ} 31^{\prime} \mathrm{N}-44^{\circ} 40^{\prime} \mathrm{N}\right)$, China. All experimental animals were treated in accordance with the National Animal Welfare Legislation. All experimental procedures were carried out in accordance with the guidelines of Beijing Forestry University. Dorsal skin samples were obtained after anesthetized by diethylether. A portion of each skin sample was immediately fixing for $24 \mathrm{~h}$ in $4 \%$ paraformaldehyde in $0.05 \mathrm{M}$ phosphate buffered saline (PBS), $\mathrm{pH}$ 7.4, for histological and immunohistochemical analyses. The remaining skin portion was stored at $-80^{\circ} \mathrm{C}$ for protein and RNA extraction.

\section{Histology}

The skin samples were dehydrated by a certain concentration of ethanol and xylene, and immersed and embedded in paraffin [10]. Sagittal serial sections $(5 \mu \mathrm{m})$ were adhered on slides coated with ploy-l-lysine (Sigma-Aldrich, St. Louis, MO, USA). Sections were stained with hematoxylin and eosin (HE) for general histological observations.

\section{Immunohistochemistry}

After deparaffinization and rehydration, serial sections of skin tissue were washed in PBS (10 M), heated in sodium citrate buffer and allowed to cool naturally to room temperature. Sections were incubated with $10 \%$ normal goat serum to reduce background staining caused by secondary antibody. Then the sections were incubated with primary polyclonal antibody against TLR4 (1:200) (bs-20595R, Beijing Biosynthesis Biotechnology Co., China), MyD88 (1:200) (bs-1047R, Beijing Biosynthesis Biotechnology Co., China), IRAK4 (1:200) (bs-2440R, Beijing Biosynthesis Biotechnology Co., China) for $12 \mathrm{~h}$ at $4^{\circ} \mathrm{C}$. The control sections were treated with normal rabbit IgG (bs-0295G, Beijing Biosynthesis Biotechnology Co., China) instead of the primary antisera. The sections were incubated with a secondary antibody for $30 \mathrm{~min}$, goat anti-rabbit $\lg G$ conjugated with biotin and peroxidase with avidin, using a rabbit ExtrAvidin staining kit (SigmaAldrich), followed by visualizing with $30 \mathrm{mg}$ 3,3-diaminobenzidine (Wako, Tokyo, Japan) solution in 150 $\mathrm{mL}$ of $0.05 \mathrm{M}$ Tris- $\mathrm{HCl}$ buffer, plus $30 \mu \mathrm{L} \mathrm{H} 202$. Finally, the reacted sections for TLR4, MyD88 and IRAK4 were counterstained with hematoxylin solution (Merck, Tokyo, Japan).

\section{Western Blotting}

Dorsal skin was weighed and homogenized in a homogenizer containing $300 \mu \mathrm{L}$ of $10 \mathrm{mg} / \mathrm{mL}$ phenylmethanesulfonyl fluoride (PMSF) stock and incubated on ice for $30 \mathrm{~min}$ throughout all the procedures. Skin homogenates were centrifuged at $12,000 \times \mathrm{g}$ for $10 \mathrm{~min}$ at $4^{\circ} \mathrm{C}$, and the supernatant was extracted. Protein extracts $(20 \mu \mathrm{g})$ were blended with an equal volume of $2 \times$ Laemmli sample buffer. Equal amount of each sample was loaded and run on a $12 \%$ SDS-PAGE gel at $18 \mathrm{~V} / \mathrm{cm}$, and then transferred to nitrocellulose membranes using a wet transblotting apparatus for $30 \mathrm{~min}$ (BioRad, Richmond, CA, USA). The membranes were incubated in blocking solution ( $2 \% \mathrm{BSA}$ ) for $1 \mathrm{~h}$ at room temperature. Primary incubation of the membranes was carried out using a 1:800 dilution of rabbit anti-rat TLR4, MyD88 and IRAK4, which were the same antibodies as used in immunohistochemistry at $4{ }^{\circ} \mathrm{C}$ overnight. Secondary incubation of the membranes was carried out using a 1:1000 dilution of goat anti-rabbit or anti-mouse IgG tagged with horseradish peroxidase for $1 \mathrm{~h}$. Finally, the membrane was stained with $10 \mathrm{mg} 3$, 3-diaminobenzidine (Wako) solution in $50 \mathrm{~mL}$ phosphate buffer $(0.03 \mathrm{M})$ plus $3 \mu \mathrm{L} \mathrm{H}_{2} \mathrm{O}_{2}$ and $\beta$-actin was used for the endogenous control. Pre-absorptions of the antibodies were performed with an excess of relative antigens (Sigma Chemical Co.) for the negative control. The intensities of the bands were quantified using Quantity One software (ver. 4.5, Bio-Rad Laboratories, Shanghai, China). Quantity One software (version 4.5, Bio-Rad Laboratories, Shanghai, China) was used to quantify the bands intensity.

\section{Real-time PCR Quantification}

The mRNA expressions of TLR4, MyD88 and IRAK4 during the breeding and pre-hibernation periods were analyzed by real- 
time PCR using one-step SYBR PrimeScript RT-PCR kit (TaKaRa Company, Dalian, China). Tissues dissected from 3 to 10 individuals were pooled from Rana dybowskii to analyze expression in the skin. The primers for real-time PCR analysis were designed using the Primer 3 program (Table 1). The PCR reactions were carried out in a $20 \mu \mathrm{L}$ volume and performed with ABI PRISM 7500 Fast Real-Time PCR System (Applied Biosystems, Foster City, CA, USA) using the following conditions: reverse transcription at $42^{\circ} \mathrm{C}$ for 5 min and $95^{\circ} \mathrm{C}$ for $30 \mathrm{~s}$, followed by PCR reaction of 40 cycles at $95^{\circ} \mathrm{C}$ for $30 \mathrm{~s}, 60^{\circ} \mathrm{C}$ for $30 \mathrm{~s}$ and $70^{\circ} \mathrm{C}$ for $30 \mathrm{~s}$ and dissociation protocol. Transcript levels of the target genes were normalized to the $\beta$-actin after correcting for differences in amplification efficiency. The expression level of each target mRNA relative to $\beta$-actin mRNA was determined using the 2- $\Delta \Delta \mathrm{Ct}$ method.

Table 1: Oligonucleotide primers used for quantitative real-time PCR.

\begin{tabular}{|c|c|c|}
\hline Gene & Sequence of Primer & Product Size (bp) \\
\hline TLR4 & $\begin{array}{c}\text { CGTCAGATGTACTCCACC (forward) } \\
\text { GAAACTCAATGAGATCTGC (reverse) }\end{array}$ & 133 \\
\hline MyD8 & $\begin{array}{l}\text { CGCCACCGTTACAAGTTGAA (forward) } \\
\text { AATGCATCAAACAGCTCGGG (reverse) }\end{array}$ & 100 \\
\hline IRAK & $\begin{array}{l}\text { GCTGCTTCTCTTCTGCTTCC (forward) } \\
\text { GCTGCTGACATCTCACATGG (reverse) }\end{array}$ \\
\hline
\end{tabular}

\section{Statistical Analysis}

Statistical comparisons were made with Student's t-test using GraphPad Prism 6. A value of $\mathrm{P}<0.05$ was considered statistically significant.

\section{Results}

\section{Histological Structure of Rana dybowskii Skin}

The observation of the structure of the Rana dybowskii dorsal skin during the breeding and pre-hibernation periods was shown in Figure 1a \& 1b. The skin of Rana dybowskii consisted of the epidermis and dermis. The epidermis consisted of stratum corneum, stratum granulosum, stratum spinosum and stratum germinativum. The dermis was composed of stratum spongiosum and stratum compactum. Mucous and granular glands are localized to the upper region of the dermis (stratum spongiosum). (Figure 1a \& 1b). The stratum compactum consisted essentially of collagen fibers arranged in a closely parallel wavy shape. Pigment cells were located between the epidermis and the dermis, accumulating to plaque during the breeding period, and dispersing in prehibernation (Figure 1c \& 1d).

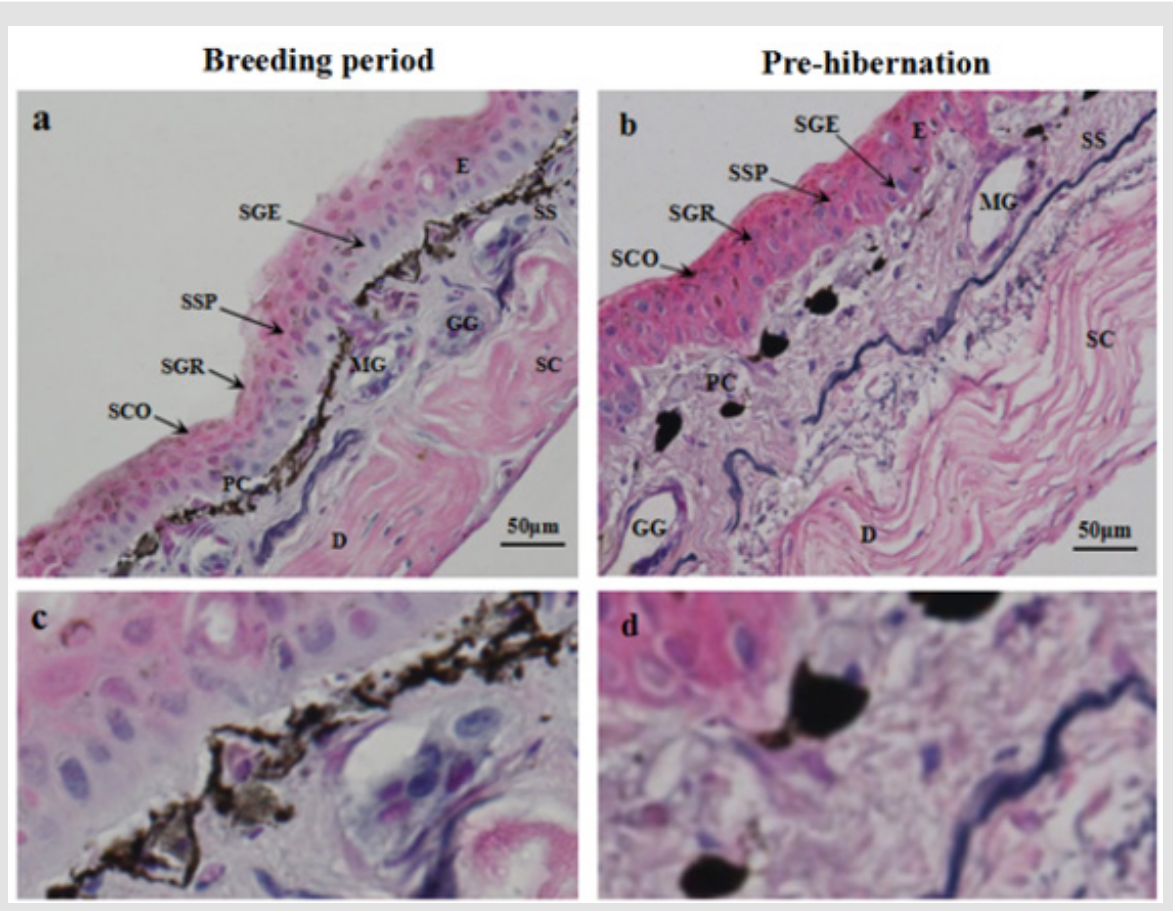

Figure 1: Structure observations of the skin from Rana dybowskii during the breeding period (a) and pre-hibernation (b). Pigment cells (PC) in the dermis during the breeding period (c) and pre-hibernation (d). E, epidermis; D, dermis; SCO, stratum corneum; SGR, stratum granulosum; SSP, stratum spinosum; SGE, stratum germinativum; SS, spongy layer; SC, dense layer; PC, pigment cells; MG, mucus glands; GG, granular glands. 


\section{Immunolocalizations of TLR4, MyD88 and IRAK4 in Rana dybowskii Skin}

The immunolocalizations of TLR4, MyD88 and IRAK4 were detected in the skin of Rana dybowskii during the breeding and prehibernation periods (Figure 2). TLR4 (Figure 2a \& 2b) and MyD88
(Figure 2c \& 2d) were localized in epidermal cells and glandular cells of the breeding and pre-hibernation periods. In addition, IRAK4 was mainly localized to glandular cells in the breeding and pre-hibernation periods, and the positive signal in epithelial cells was weak (Figure 2e \& 2f).

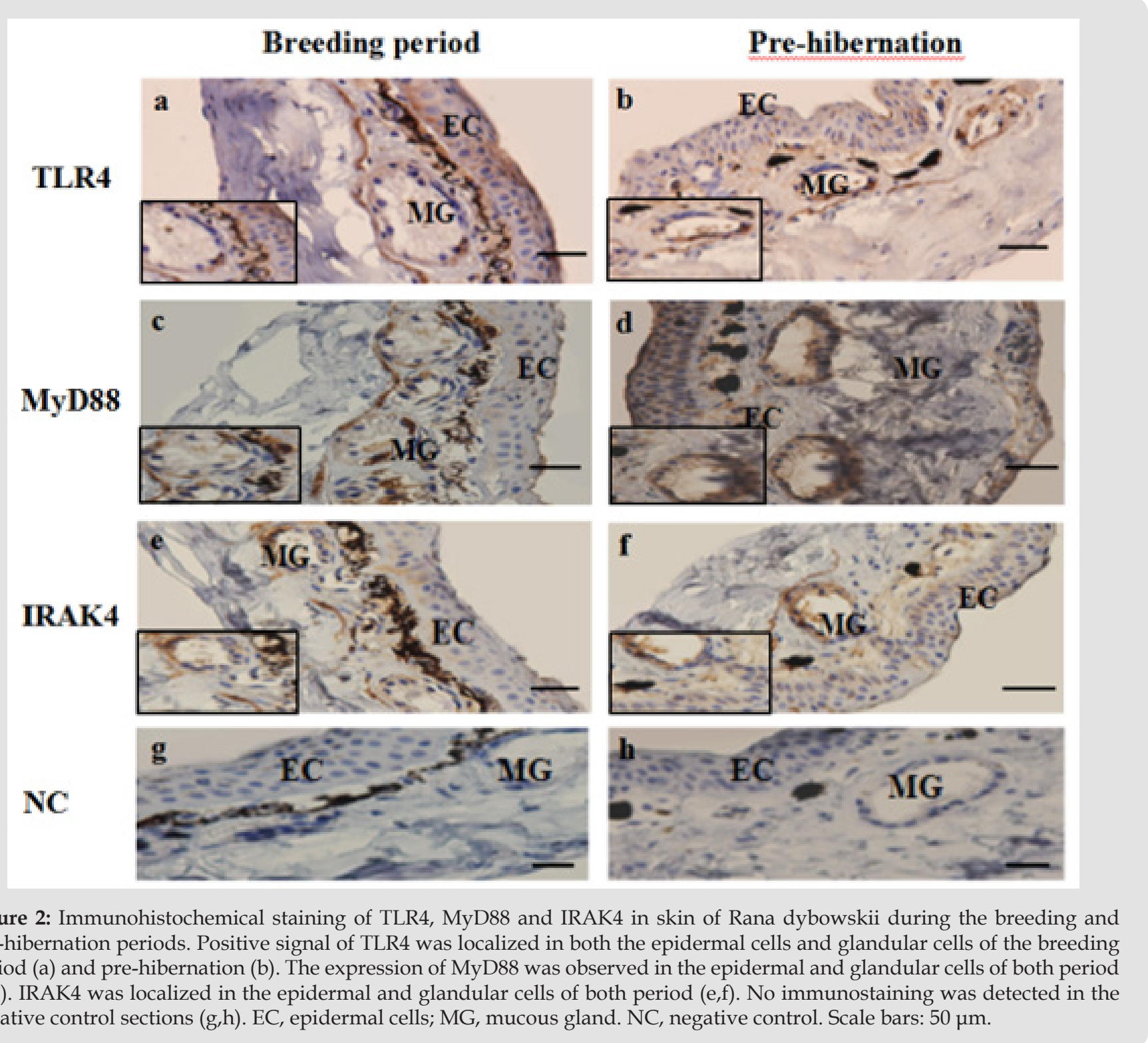

\section{The Expressions of TLR4, MyD88 and IRAK4 in Rana dybowskii Skin}

The results of Western blot analysis of TLR4, MyD88 and IRAK4 in the dorsal skin of Rana dybowskii in the breeding and pre-hibernation periods were shown in Figure 3. The bands of approximately $90 \mathrm{kDa}, 34 \mathrm{kDa}$ and $51 \mathrm{kDa}$ represented TLR4 (Figure 3a), MyD88 (Figure 3b) and IRAK4 (Figure 3c), respectively. Quantification was normalized to expression of the endogenous control $\beta$-actin. The results showed that the protein expressions of TLR4, MyD88 and IRAK4 were present in the skin, but there were no significant differences in protein expression levels between the two periods (Figure 3a-3c). Primary antibody pre-absorbed with excess antigen was used as a negative control (Figure 3 lane NC).

The mRNA levels of TLR4, MyD88 and IRAK4 were detected in the skin tissues of Rana dybowskii during the breeding and prehibernation periods (Figure 4). The results showed that the relative mRNA level of MyD88 in pre-hibernation decreased compared with that of the breeding period (Figure 4b), while the mRNA level of TLR4 and IRAK4 did not differ significantly between the two periods (Figure 4a, c). 


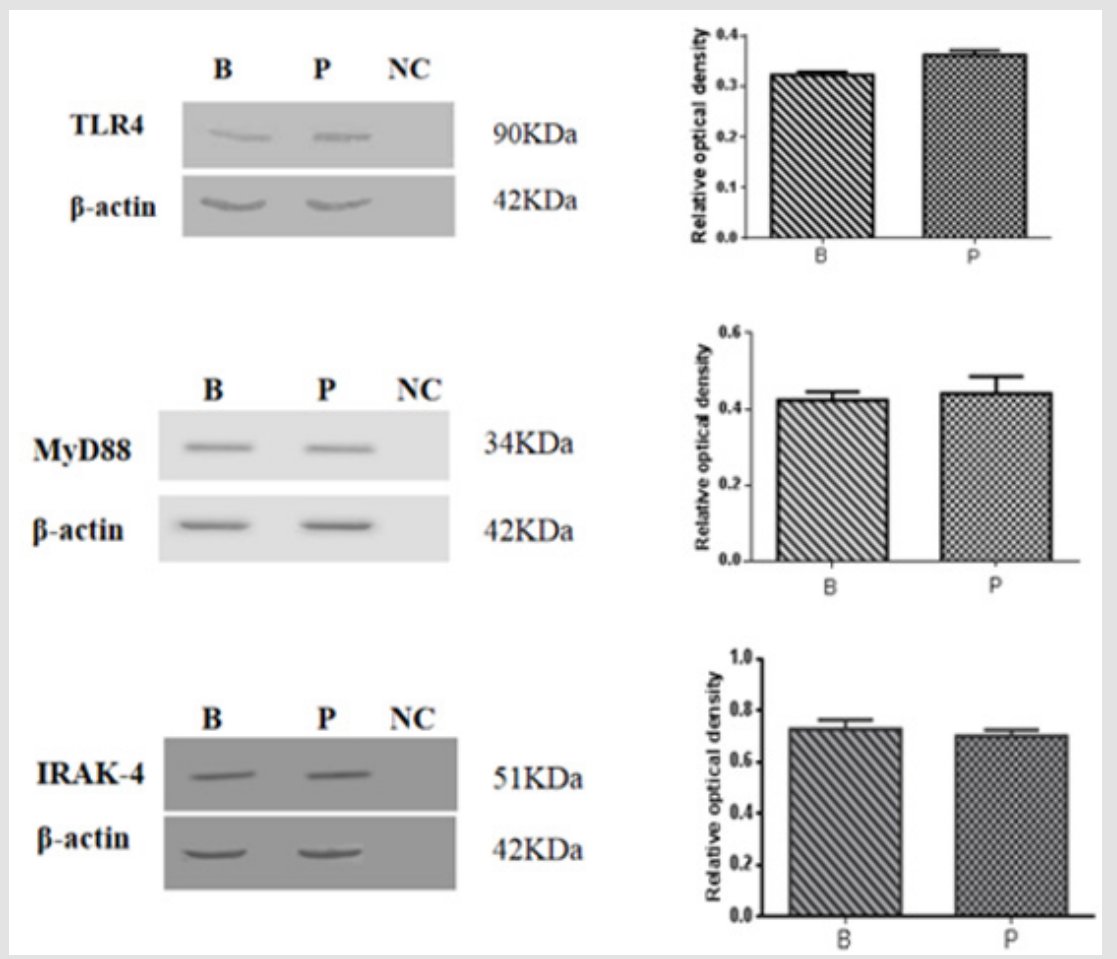

Figure 3: Western blot analysis of TLR4, MyD88 and IRAK4 in the dorsal skin of Rana dybowskii during the breeding (B) and pre-hibernation $(\mathrm{P})$ periods. The band of TLR4 (a) is approximately $90 \mathrm{kDa}$, the MyD88 (b) is approximately $34 \mathrm{kDa}$, and the band of IRAK4 (c) is approximately $51 \mathrm{kDa}$. A pre-absorbed primary antibody was used instead of the primary antibody for the negative control (lane NC). The level of expression was determined by densitometry. Bars represent the mean \pm SD of three independent experiments. B, the breeding period; $\mathrm{P}$, pre-hibernation.

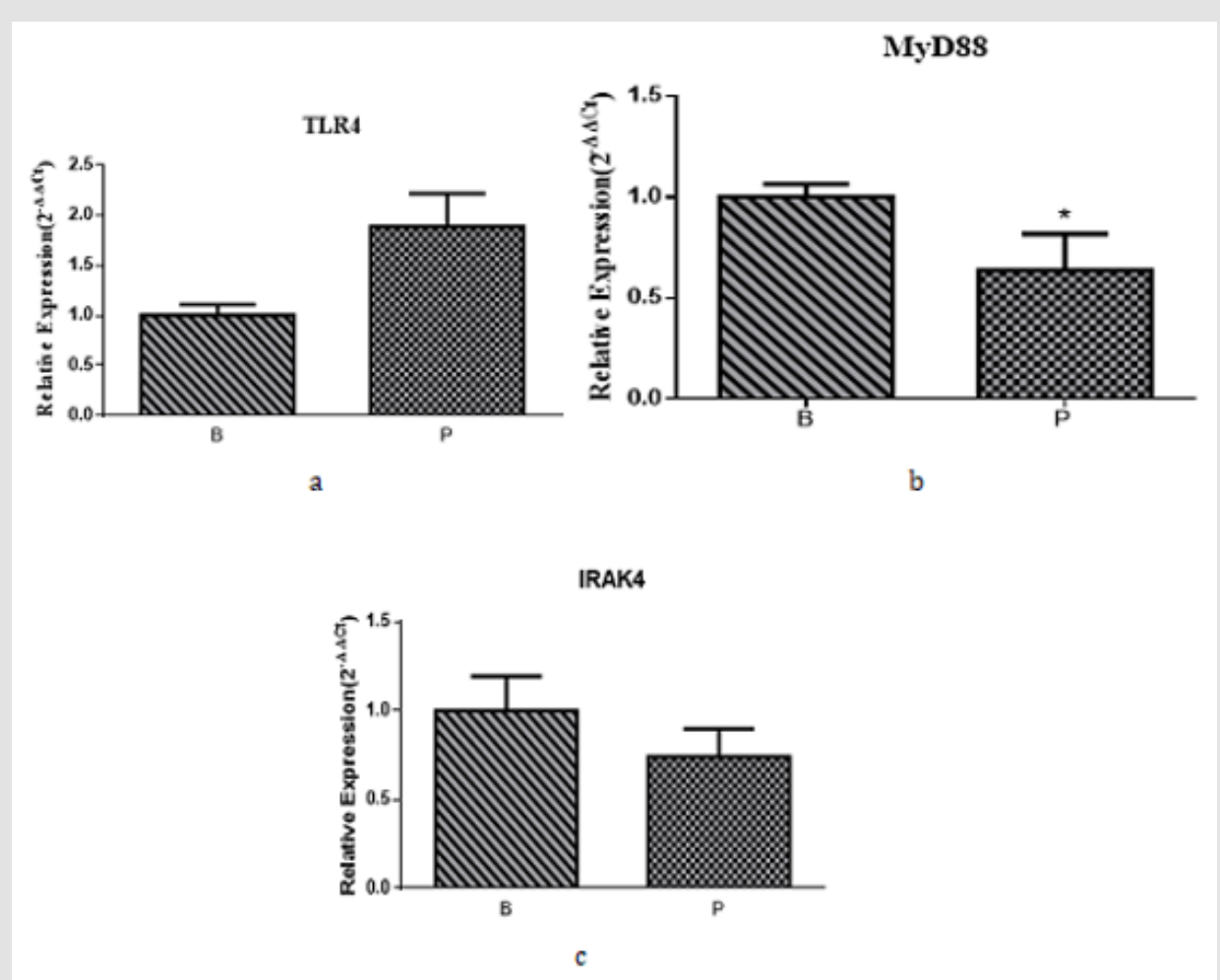

Figure 4: Real-time PCR results of TLR4, MyD88 and IRAK4 in the dorsal skin of Rana dybowskii during the breeding and pre-hibernation periods. The relative mRNA levels of TLR4, MyD88 and IRAK4 in Rana dybowskii in the breeding and prehibernation periods skin were shown in (a), (b) and (c), respectively. Bars represent the mean \pm SD of three independent experiments. $\mathrm{B}$, the breeding period; $\mathrm{P}$, pre-hibernation; ${ }^{*} \mathrm{P}<0.05$. 


\section{Discussion}

The present results demonstrated that TLR4, MyD88 and IRAK4 were localized in epithelial and gland cells of the skin of Rana dybowskii in the breeding and pre-hibernation periods. The mRNA expression levels of TLR4 and IRAK4 were not significantly different in two periods, while the mRNA expression level of MyD88 in pre-hibernation was lower than that of the breeding period. In addition, the protein expression levels of TLR4, MyD88 and IRAK4 did not differ between the breeding and pre-hibernation periods. These findings suggested that TLR4, MyD88 and IRAK4 might be involved in the regulation of Rana dybowskii skin immune function during the breeding and pre-hibernation periods.

Amphibian skin glands are basically composed of two types: mucus glands and granular glands. The mucous glands secrete mucus to keep it moist and prevent mechanical damage to delicate skin and delay evaporation of water [14]. Granular glands could synthesize a variety of chemicals such as peptides and alkaloids, to provide protection against fungal and bacterial infections and to resist predators [15]. In addition, glandular secretions are closely related to various biological effects such as cytotoxic, bactericidal, fungicidal, lytic, anesthetic, and phenomenal [16]. One previous report has shown that proteins in the skin mucus of Chinese giant salamander participated in the physiological activities of the skin through various ways, including defense, immune response, wound healing and breathing [16]. Previous studies have shown that inflammatory factors such as IL-1 $\beta$, IL-1R, IL- 6 and TNF- $\alpha$ were expressed in the skin of Rana dybowskii, indicating that these cytokines might be involved in skin immune and inflammatory responses against external aggressors $[17,11]$. The present study further confirmed that TLR4, MyD88 and IRAK4 were expressed in the glandular cells of Rana dybowskii skin during the breeding and pre-hibernation periods, implying that inflammatory factors might be activated by the TLR4 signaling pathway of the glandular cells themselves. And the granular glands might play an important role in the immune function of the skin in Rana dybowskii by regulating immune-related proteins and cytokines.

The TLR4 signaling pathway is an important pathway mediating inflammatory responses. When inflammation occurs in the body, TLR4 binds to the pathogen-associated ligand on the cell membrane and activates the immune response [18]. The previous study has shown that TLR4 was expressed in skin fibroblasts and the activation of NF- $\kappa$ B was induced after treatment of skin fibroblasts with the corresponding ligands [19]. These results indicated that TLR4 could respond functionally to its ligand, suggesting that TLR4 in skin fibroblasts plays a crucial role in the detection and response of different types of pathogens or risk signals. In this study, we demonstrated that TLR4 was expressed in Rana dybowskii skin during the breeding and pre-hibernation periods. Our previous study also showed that NF- $\mathrm{KB}$ was also expressed in the skin of Rana dybowskii [13]. Combining those evidence, our current results supported the view that TLR4 played a role in the immune defense function of Rana dybowskii skin by identifying MyD88 to activate the downstream signaling pathway, such as transcription factor NF- $\kappa B$, causing the immune response [20].

As a universal adaptor protein, MyD88 regulates most TLR and interleukin-1 receptor (IL-1R) signaling as well as immune responses against viral and bacterial infections [21]. In the MyD88-dependent pathway, MyD88 recruits IRAKs, and IRAK4 is first activated, thereby activating downstream genes in the TLR signaling pathway, such as NF- $\mathrm{KB}$, IRF3 or IRF7, and finally inducing the production of pro-inflammatory cytokines such as interleukin (IL), tumor necrosis factor (TNF) and type I interferon (IFN), these factors could mediate direct defense responses and modulate immune function [22,23]. The present results directly demonstrated that MyD88 was expressed in the skin of Rana dybowskii during the breeding and pre-hibernation periods. Although the mRNA expression level of MyD88 in pre-hibernation was lower than that of the breeding period, there was no significant difference in the expression of MyD88 between the two stages, indicating that MyD88 did not show seasonal expression in Rana dybowskii skin. It was speculated that the TLR4/MyD88 signaling pathway might be indispensable in the immune defense function of Rana dybowskii skin.

IRAK4 is a downstream adapter molecule of MyD88 that is critically required for the induction of many TLR/IL-1R-dependent immune responses [24]. Endogenous IRAK4 interacts with IRAK1 and TRAF6 in an IL-1-dependent manner, and its overexpression induces activation of NF- $\mathrm{B}$ and mitogen-activated protein (MAP) kinase pathways, which in turn causes inflammation and immune responses [25]. Moreover, defects in IRAK4 would cause severe TLR signaling and NF- $\kappa B$ signaling, suggesting that IRAK4 plays an important role in TLR-mediated pathways [26,27]. Our results showed that the protein and molecular expression of IRAK4 were present in the skin of Rana dybowskii during the breeding and prehibernation periods, indicating that the skin of Rana dybowskii could produce IRAK4. The presence of TLR4, MyD88 and IRAK4 in the same types of cells including epithelial and glandular cells, indicating the complete TLR4/MyD88/IRAK4 signaling pathway in the skin of Rana dybowskii. In addition, the positive signal of IRAK4 were weak in the epithelial cell comparing with that in the glandular cell indicated the stronger TLR4/MyD88/IRAK4 signaling in the glandular cells which might be due to the direct contact to the external environment in the glandular duct and easy for the pathogen invasion.

In summary, our previous studies have shown that IL-1 $\beta$, IL-1R, IL-6, TNF- $\alpha$ and NF- $\kappa$ B were found in the skin of Rana dybowskii, and these cytokines may be involved in immune and inflammatory responses $[17,11]$. The present study demonstrated that TLR4, MyD88 and IRAK4 were expressed in Rana dybowskii skin during the breeding and pre-hibernation periods. These results suggested that TLR4 might regulate the secretion of these cytokines via the MyD88 signaling pathway, which plays a regulatory role in the 
immune response of Rana dybowskii skin. The data presented here will greatly aid the dissection of TLR4 signaling pathway in the skin of amphibians.

\section{Acknowledgments}

This research work is supported by Beijing Natural Science Foundation (8182039) and National Natural Science Foundation of China $(31872320,21806010)$ and Young Scientist Start-up funding of Beijing Forestry University (BLX201714).

\section{References}

1. Yu L, Y Heng, Z Ming, L Qianjin (2014) TLR2 and TLR4 in autoimmune diseases: a comprehensive review. Clin Rev Allergy Immunol 47(2): 136147.

2. Iotzova-Weiss G, SN Freiberger, P Johansen, J Kamarachev, E Guenova, et al. (2017) TLR4 as a negative regulator of keratinocyte proliferation. PLoS One 12(10): e0185668.

3. Tao Y, Y Wang, X Wang, C Wang, K Bao, et al. (2017) Calycosin Suppresses Epithelial Derived Initiative Key Factors and Maintains Epithelial Barrier in Allergic Inflammation via TLR4 Mediated NF-кB Pathway. Cell Physio Biochem 44(3): 1106-1119.

4. Wang L, K Yu, X Zhang, S Yu (2018) Dual functional roles of the MyD88 signaling in colorectal cancer development. Biomed Pharmacother 107: 177-184.

5. Palti Y (2011) Toll-like receptors in bony fish: From genomics to function. Dev Comp Immunol 35(12): 1263-1272.

6. Costa S, O Marini, D Bevilacqua, AL DeFranco, B Hou, et al. (2017) Role of MyD88 signaling in the imiquimod-induced mouse model of psoriasis: focus on innate myeloid cells. J Leukoc Biol 102(3): 791-803.

7. Sabine H, MK Oyoshi, JL Hornick, RS Geha (2018) MyD88 signaling in T regulatory cells by endogenous ligands dampens skin inflammation in filaggrin deficient mice. Clin Immunol 195: 88-92.

8. Nobutaka S, S Shinobu, GS Duncan, DG Millar, W Teiji, et al. (2002) Severe impairment of interleukin-1 and Toll-like receptor signalling in mice lacking IRAK-4. Nature 416: 750-756.

9. Xueqing $X, L$ Ren (2015) The chemistry and biological activities of peptides from amphibian skin secretions. Chem Rev 115: 1760-1846.

10. Pfützner W, J Gutermuth (2014) Das Immunsystem der Haut. Allergo Journal 23(3): 16-17.

11. Xi L, W Chen, P Chen, Y Qi, R Hu, et al. (2017a) Expressions of IL-6, TNF- $\alpha$ and NF-KB in the skin of Chinese brown frog (Rana dybowskii). Eur ] Histochem 61(4): 2834.

12. Xiang-Hong X, M Hui-Min, X Yi-Gang, Z Jing-Yu, C Long-Hui, et al. (2014) Analysis of skin and secretions of Dybowski's frogs (Rana dybowskii) exposed to Staphylococcus aureus or Escherichia coli identifies immune response proteins. Vet J 200(1): 127-132.

13. Niu S, X Shi, J Zhang, L Chai, X Xiao (2016) Cloning, Characterization, and Expression Analysis of MyD88 in Rana dybowskii. Appl Biochem Biotechnol 179(2): 294-306.

14. Clarke BT (1997) The natural history of amphibian skin secretions, their normal functioning and potential medical applications. Biol Rev 72(3): 365-379.

15. Wanninger M, T Schwaha, E Heiss (2018) Form and Function of the skin glands in the Himalayan newt Tylototriton verrucosus. Zoological Lett 4: 15
16. Guo W, M Ao, W Li, J Wang, L Yu (2012) Major biological activities of the skin secretion of the Chinese giant salamander, Andrias davidianus. Z Naturforsch C 67(1-2): 86-92.

17. Xi L, R Hu, T Guo, Y Wang, X Sheng, et al. (2016) Immunoreactivities of NF-KB, IL-1 $\beta$ and IL-1R in the skin of Chinese brown frog (Rana dybowskii). Acta Histochem 119(1): 64-70.

18. Kawai T, S Akira (2011) Toll-like Receptors and Their Crosstalk with Other Innate Receptors in Infection and Immunity. Immunity 34(5): 637-650.

19. Yao C, JH Oh, DH Lee, JS Bae, CL Jin, et al. (2015) Toll-like receptor family members in skin fibroblasts are functional and have a higher expression compared to skin keratinocytes. Int J Mol Med 35(5): 1443-1450.

20. Feng H, R Su, Y Song, C Wang, L Lin, et al. (2016) Positive Correlation between Enhanced Expression of TLR4/MyD88/NF- $\mathrm{KB}$ with Insulin Resistance in Placentae of Gestational Diabetes Mellitus. PLoS One 11(6): e0157185.

21. Dai C, L Sun, L Yu, G Zhu, S Wu, et al. (2016) Effects of Porcine MyD88 Knockdown on the Expression of TLR4 Pathway-related Genes and Proinflammatory Cytokines. Biosci Rep 36(6).

22. Kang Y, G Su, J Sun, Y Zhang (2018) Activation of the TLR4/MyD88 signaling pathway contributes to the development of human hepatocellular carcinoma via upregulation of IL-23 and IL-17A. Oncol Lett 15(6): 9647-9654.

23. Kim S, J Bang, C Son, W Baek, J Kim (2018) Grape seed proanthocyanidin extract ameliorates murine autoimmune arthritis through regulation

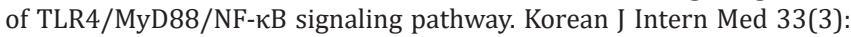
612-621.

24. Gosu V, S Basith, P Durai, S Choi (2012) Molecular evolution and structural features of IRAK family members. PLoS One 7(11): e49771.

25. Shyun L, S Astrid, EJ Fontana, W Holger (2002) IRAK-4: a novel member of the IRAK family with the properties of an IRAK-kinase. Proc Natl Acad Sci U S A 99(8): 5567-5572.

26. Capucine P, P Anne, B Marion, K Cheng-Lung, B Jacinta, et al. (2003) Pyogenic bacterial infections in humans with IRAK-4 deficiency. Science 299: 2076-2079.

27. Paciolla M, A Pescatore, MI Conte, E Esposito, M Incoronato, et al. (2015) Rare mendelian primary immunodeficiency diseases associated with impaired NF-kB signaling. Genes Immun 16(4): 239-246.

28. Brown J, H Wang, GN Hajishengallis, M Martin (2011) TLR-signaling networks: an integration of adaptor molecules, kinases, and cross-talk. J Dent Res 90(4): 417-427.

29. Martin LB, ZM Weil, R J Nelson (2008) Seasonal changes in vertebrate immune activity: mediation by physiological trade-offs. Philos Trans $R$ Soc Lond B Biol Sci 363(1490): 321-339.

30. Sherwani MA, K Yang, A Jani, RA Abed, AK Taufique, et al. (2019) Protective Effect of Baicalin Against TLR4-mediated UVA-induced Skin Inflammation. Photochem Photobiol 95(2): 605-611.

31.Xi L, Y Liu, Z Tang, X Sheng, H Zhang, et al. (2017b) Expression of leptin receptor in the oviduct of Chinese brown frog (Rana dybowskii). Am J Physiol Regul Integr Comp Physiol 312(6): R912-R918.

32. Xu YG, LH Chai, W Shi, DD Wang, JY Zhang, et al. (2017) Transcriptome profiling and digital gene expression analysis of the skin of Dybowski's frog (Rana dybowskii) exposed to Aeromonas hydrophila. Appl Microbiol Biotechnol 101(14): 5799-5808.

33. Yoo I, J Han, S Lee, W Jung, J H Kim, et al. (2019) Analysis of stage-specific expression of the toll-like receptor family in the porcine endometrium throughout the estrous cycle and pregnancy. Theriogenology 125: 173183. 


\section{ISSN: 2574-1241}

DOI: $10.26717 /$ BJSTR.2019.23.003979

Meiyu Xu. Biomed J Sci \& Tech Res

\section{(c) (P) This work is licensed under Creative}

Submission Link: https://biomedres.us/submit-manuscript.php

$\begin{array}{ll}\text { BIOMEDICAL } & \text { Assets of Publishing with us } \\ \text { RESEARCHES } & \text { - Global archiving of articles } \\ & \text { - Immediate, unrestricted online access } \\ & \text { - Rigorous Peer Review Process } \\ \end{array}$

